Canadian

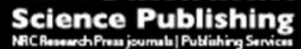

Canadian Journal of Physiology and Pharmacology Revue canadienne de physiologie et pharmacologie

\title{
The effects of motor rehabilitation training on clinical symptoms and serum BDNF levels in Parkinson's disease subjects
}

\begin{tabular}{|r|l|}
\hline Journal: & Canadian Journal of Physiology and Pharmacology \\
\hline Manuscript ID & cjpp-2015-0322.R2 \\
\hline Danuscript Type: & Article \\
\hline Complete List of Authors: & $\begin{array}{l}\text { Angelucci, Francesco; IRCCS Santa Lucia Foundation, Department of } \\
\text { Clinical and Behavioral Neurology } \\
\text { Piermaria, Jacopo; IRCCS Santa Lucia Foundation, Department of Clinical } \\
\text { and Behavioral Neurology, } \\
\text { Gelfo, Francesca; IRCCS Santa Lucia Foundation, ; } \\
\text { Shofany, Jacob; IRCCS Santa Lucia Foundation, Department of Clinical and } \\
\text { Behavioral Neurology, } \\
\text { Tramontano, Marco; IRCCS Santa Lucia Foundation, Department of Clinical } \\
\text { and Behavioral Neurology, } \\
\text { Fiore, Marco; IBCN-CNR, Institute of Cell Biology and Neurobiology } \\
\text { Caltagirone, Carlo; IRCCS Santa Lucia Foundation, } \\
\text { Peppe, Antonella; IRCCS Santa Lucia Foundation, }\end{array}$ \\
\hline Keyword: & \multicolumn{2}{|c|}{} \\
\hline
\end{tabular}




\section{The effects of motor rehabilitation training on clinical symptoms and serum BDNF levels in Parkinson's disease subjects}

Francesco Angelucci ${ }^{a^{*}}$, Jacopo Piermaria ${ }^{\mathrm{a}}$, Francesca Gelfo ${ }^{\mathrm{a}, \mathrm{b}}$, Jacob Shofany ${ }^{\mathrm{a}}$, Marco Tramontano ${ }^{\mathrm{a}}$, Marco Fiore $^{\mathrm{c}}$, Carlo Caltagirone ${ }^{\mathrm{a}, \mathrm{b}}$, Antonella Peppe ${ }^{\mathrm{a}}$

${ }^{a}$ Department of Clinical and Behavioural Neurology, IRCCS Santa Lucia Foundation, Via Ardeatina 354, 00142, Rome, Italy

${ }^{b}$ Department of Systemic Medicine, "Tor Vergata” University, Via Montpellier 1, 00133, Rome, Italy Institute of Cellular Biology and Neurobiology. CNR, Via del Fosso di Fiorano 64, 00143 Rome, Italy

*Corresponding author:

Francesco Angelucci

IRCCS Santa Lucia Foundation

Department of Clinical and Behavioral Neurology

00179, Rome, Italy

Phone: 0039-06-51501550

Fax: 0039-06-51501552

e-mail address: f.angelucci@hsantalucia.it 


\begin{abstract}
Increasing evidence suggests that motor rehabilitation may delay Parkinson's disease (PD) progression. Moreover, parallel treatments in animals up-regulate brain-derived neurotrophic factor (BDNF). Thus, we investigated the effect of a motor rehabilitation protocol on PD symptoms and BDNF serum levels. Motor rehabilitation training consisted of a cycle of 20 days/month of physiotherapy divided in three daily sessions. Clinical data were collected at the beginning, at the end and at 90 days follow-up. BDNF serum levels were detected by ELISA at 0, 7, 14, 21, 30 and 90 days. The follow up period had a duration of 60 days (T30-T90). The results showed that at the end of the treatment (day $30^{\text {th }}$ ), an improvement in extrapyramidal signs (UPDRS III; UPDRS III - Gait and Balance items), motor (6 Minute Walking Test) and daily living activities (UPDRS II; PDQ-39) was observed. BDNF levels were increased at day $7^{\text {th }}$ as compared to baseline. After that, no changes in BDNF were observed during the treatment and in the successive follow-up. This study demonstrates that motor rehabilitation training is able to ameliorate PD symptoms and to increase temporarily BDNF serum levels. The latter effect may potentially contribute to the therapeutic action.
\end{abstract}

Key words: Motor rehabilitation, physical exercise; BDNF; Parkinson's disease 


\section{INTRODUCTION}

Parkinson's disease (PD) is a chronic neurodegenerative disease leading to motor dysfunctions characterized by resting tremor, bradykinesia, rigidity and postural instability (Gazewood et al. 2013). PD is caused by the progressive loss of dopaminergic neurons in the midbrain substantia nigra pars compacta, resulting in reduced dopamine level in the striatum (Ali et al. 2011). Unfortunately, pharmacological treatments are able to reduce the symptoms, but do not block the disease progression (Worth 2013) and, on the long term, produce significant clinical motor and no motor side effects such as dyskinesia (Huot et al.2013) and impulse control disorders (Garcia-Ruiz et al. 2014; Sharma et al. 2015; Weintraub et al. 2009). For these reasons, in the recent years the scientific community has started to explore alternative non pharmacological therapeutic approaches.

Among them, therapeutic strategies based on physical rehabilitation have generated considerable interest in view of the fact that physical exercise may slow PD progression (Frazzitta et al. 2013; Mehrholz et al. 2010; Tomlinson et al. 2013). Particularly, preliminary data indicate that the natural worsening of symptoms associated with PD can be contrasted by rehabilitation protocols based on physical therapy, in association with treadmill training and auditory and visual cues, thus reducing the need for increasing levodopa (L-dopa) doses (Frazzitta et al. 2010). Nonetheless, despite these positive results, the mechanisms of action of these treatments and/or the biological correlates involved are still under investigation.

Interestingly, parallel treatments in animal models, such as physical exercise and environmental enrichment, may upregulate members of the neurotrophic factor family, including brain-derived neurotrophic factor (BDNF) (Bekinschtein et al. 2011; Gelfo et al. 2011). and glial cell-derived neurotrophic factor (GDNF) (McCullough et al .2013). BDNF is a potent trophic factor for the dopaminergic neurons of the substantia nigra (Hyman et al. 1991) and its involvement in PD is widely documented in animal models and humans. In rodent models, it has been demonstrated that fibroblasts 
genetically engineered to produce BDNF prevented the loss of dopaminergic neurons in the substantia nigra after 6-hydroxydopamine or 1-methyl-4-phenylpyridinium (MPP)-induced lesion (Frim et al. 1994; Levivier et al. 1995). Moreover, direct BDNF infusion in the brain of non-human primates has also shown to produce beneficial effects in the MPP experimental model by reducing cell loss and enhancing striatal innervations (Tsukahara et al. 1995). In PD patients, expression of BDNF protein and mRNA is reduced in nigral neurons (Mogi et al. 1999; Parain et al. 1999), together with reduced BDNF serum levels (Ricci et al. 2010; Scalzo et al. 2010).

These data suggest that BDNF may potentially represent a component of the mechanism of action of physical rehabilitation in PD. This is supported by data showing that BDNF peripheral levels may increase after physical exercise (Schmolesky et al. 2013; Vaughan et al. 2014). In addition, it was demonstrated that the increase in BDNF after physical training correlates with improvements in cognitive functions in aged non pathological subjects (Vaughan et al. 2014) as well as in patients affected by pathological conditions such as stroke (El-Tamawy et al. 2014) and depression (Pereira et al. 2013; Oral et al. 2012).

Thus, the aim of this study was to explore the relationship between the effects of a motor rehabilitation program (4-week cycle), consisting of a session of standard physical therapy and a session of treadmill and Wii Fit balance board training, and the changes in BDNF serum levels in a group of PD patients.

\section{METHODS}

\section{Subjects}

Nine (2 women, 7 men) rigid-akinetic subjects with a diagnosis of Parkinson's disease (Hoehn \&Yahr: II-III) were included in the study (Table 1). The diagnosis of PD was made by an expert neurologist according to the United Kingdom Parkinson's Disease Society brain bank criteria and based on the 
presence of at least two of the four cardinal symptoms and a good chronic response to L-dopa treatment (Gelb et al. 1999).

The demographic and clinical characteristics at the time of the study were as follow: age $(62.78 \pm 6.68$ years); PD age onset (51 \pm 11.40 years); years of disease (11.78 \pm 7.38$)$; years of L-dopa treatment (10.56 \pm 7.21$)$; present L-dopa dosage $(570.06 \pm 267.31 \mathrm{mg})$; prevalent symptom side (7 right side/ 2 left side).

All PD subjects, hospitalized at Neurorehabilitation Unit of IRCCS Santa Lucia Foundation, were stable responders to monotherapy with L-dopa (mean daily dosage: $570.06 \pm 267.31 \mathrm{mg}$ SD + peripheral inhibitor)and they could move independently without important fall risk. The optimal range of L-dopa was evaluated during the 4 weeks preceding the training. The dose was maintained constant for at least 30 days preceding the study, during the entire experimental period and in the successive follow up. Subjects were hospitalized for the entire duration of motor rehabilitation (30 days).All patients did not show acute or chronic psychiatric symptoms at the time of the study, as evaluated by DSM IV.

Subjects gave their written consent before participation in the study, which was approved by Local Ethic Committee (Prog. 299.04/29/2011).Table 1shows demographic and clinical characteristics at the beginning of the study. An overview of the experimental design is presented in Figure 1.

\section{Inclusion criteria}

Inclusion criteria were the following: (i) absence of systemic and metabolic diseases; (ii) absence of uncertain and unclear history of chronic L-dopa treatment responsiveness; (iii) absence of brain lesions and/or marked cortical and subcortical atrophy on computed tomography (CT) and magnetic resonance (MR) scans; (iv) absence of cognitive impairment with a Mini Mental State Examination score $<24$ (Folstein et al. 1975); (V) pharmacological treatment only with L-dopa. 


\section{Motor rehabilitation training (MRT)}

MRT consisted of 4-week cycle of physiotherapy that entailed three daily sessions (2 sessions in the morning and 1 in the afternoon), 5 days a week (see Peppe et al. 2007 for further details on rehabilitation program).

The first session comprised the following types of exercises:

(a) Exercises to promote relaxation and awareness of using the diaphragm in respiration; (b) Exercises to promote neck flexibility; (c) Segmental exercises of active or assisted mobilization (flexo-extension, pronosupination) to increase strength, motility, and coordination of four limbs; (d) Exercises to improve equilibrium (in quadrupedic position); (e) Exercise to improve control of posture in different positions; (f) Training exercises for walking on level ground or between parallel bars.

The second session was subdivided into:

(a) 20 min on treadmill with a heart reserve $\leq 60 \%$ and maximum speed of treadmill scrolling of 3.5-4 $\mathrm{Km} / \mathrm{h}$; (b) $20 \mathrm{~min}$ on stationary bike with maximum speed of 25-30 Km/h, and (c) 20 min on Wii Fit balance board (WFBB) (Pompeu et al. 2012) using games in which arms and legs coordination as well as correct movements allowed to maintain balance.

The third afternoon session was a motor group therapy session in which the participants were subjected to the following exercises:

(a) Exercises to promote pectoral and hip girdle releasing; (b) Exercises to promote control of strength and movement velocity.

All subjects were able to perform the daily physiotherapy sessions and completed the motor rehabilitation program. None of them showed other clinical problems during the study time. 
The follow up period had a duration of 60 days, from the end of training (T30) until the last data collection (T90).A CD-ROM with instructions to perform exercises aimed at limb co-ordination and muscle stretching (including active or assisted limb mobilization), stimulate postural control, and exercises to improve control of posture in different positions was given to each subject to practice daily at home for at least $1 \mathrm{~h}$ per day (Peppe et al.2007). Subjects and care givers were previously trained at our Unit. The participant's adherence to the home exercises was monitored monthly by the care giver.

\section{Clinical assessments (rating scales)}

Clinical assessments, which included Unified Parkinson's Disease Rating Scale (UPDRS) part II (Activities of daily living) and III (Motor examination) (Movement Disorder Society Task Force on Rating Scales for Parkinson's Disease 2003; Vassar et al 2012), Parkinson's Disease Questionnaire (PDQ-39) (Peto et al. 1995; Zhang and Chan 2012) and 6 Minute Walking Test (6MWT) (Combs et al. 2014; Guyatt et al. 1985), were collected at the beginning (T0), at the end (T30) and at 3 months after the end of the motor rehabilitation training (T90; Figure 1).

All clinical evaluations and blood tests were carried out $2 \mathrm{~h}$ after the first morning drug administration (in ON clinical status).

\section{Blood sampling}

Blood samples were taken in the morning (8.00-9.00 a.m, to avoid circadian variations) at the beginning (T0), $7^{\text {th }}(\mathrm{T} 7), 14^{\text {th }}(\mathrm{T} 14), 21^{\text {st }}(\mathrm{T} 21)$, and $30^{\text {th }}(\mathrm{T} 30)$ days of motor rehabilitation training and at $90^{\text {th }}$ days, the end of the follow up period (T90) (Figure 1). Venous blood was collected into sampling tubes and centrifuged at $2000 \times \mathrm{g}$ for $20 \mathrm{~min}$. Serum was then aliquoted and stored at $-80{ }^{\circ} \mathrm{C}$ until analysis. Samples were collected 90 minutes after the first L-dopa daily dose and before breakfast. 


\section{Determination of BDNF content}

BDNF was detected using sandwich ELISA (R\&D Systems, USA; cat. $N^{\circ}$ DY248) according to the manufacturer's instructions. All assays were performed on F-bottom 96-well plates (Nunc, Wiesbaden, Germany). Tertiary antibodies were conjugated to horseradish peroxidase. Wells were developed with tetramethylbenzidine and measured at 450/570 $\mathrm{nm}$. BDNF content was quantified against a standard curve calibrated with known amounts of protein. The detection limit for BDNF was $15 \mathrm{pg} / \mathrm{ml}$. Measurements were performed in duplicate and values are expressed as ng/ml. Cross-reactivity to other related trophic factors (NGF, NT-3; NT-4; TGF $\beta$, TGF $\alpha$ ) was less than 3\% (Angelucci et al. 2008; Ricci et al. 2010).

\section{Statistical analysis}

The overall effects of motor rehabilitation training on clinical parameters and BDNF levels were analyzed by Friedman ANOVA. To investigate the possible short and long term changes in BDNF levels, post- hoc comparisons within logical sets of means were performed using the Wilcoxon Signed Rank test, the use of which is permissible or even recommended in absence of significant main or interaction effects in ANOVA in order to minimize frequency errors of both type I and II following the indications given by Wilcox (1987), pp. $187-189)$. $P$-values $\leq 0.05$ were considered statistically significant. Statistical evaluations were performed by SPSS 12.0 software.

\section{RESULTS}

\section{Effect of motor rehabilitation on PD rating clinical scales}

Clinical evaluations were performed at the beginning (T0) and the end (T30) and at 3 months from the end of the motor rehabilitation training (T90). 
Friedman ANOVA showed a significant main effect of the motor rehabilitation training on UPDRS II scale $(p<0.05)$. Wilcoxon Signed Rank test showed that values at T30 were significantly reduced as compared to T0 $(p<0.01)$ (Figure 2A).

Friedman ANOVAs also showed a significant main effect of the motor rehabilitation training on UPDRS III $(p<0.005)$ and UPDRS III - Gait and Balance items $(p<0.01)$. Wilcoxon Signed Rank test showed that UPDRS III and UPDRS III - Gait and Balance values at T30 $(p<0.01)$ and at T90 $(p<0.05)$ were significantly lower than those atT0 (Figure 2A).

A significant effect of the treatment on PDQ-39 was also found $(p<0.01)$ because the values at T30 $(p<0.01)$ and T90 $(p<0.01)$ were significantly lower versus T0 (Figure 2B).

Friedman ANOVA for 6MWT showed a significant effect of the treatment $(p<0.05)$. The score obtained at T30 was significantly higher than that atT0 $(p<0.01)$ (Figure $2 \mathrm{C})$.

\section{Effect of motor rehabilitation on BDNF serum levels}

Friedman ANOVA did not show an overall effect of the motor rehabilitation training on BDNF serum levels $(p<0.14)$. However, Wilcoxon Signed Rank test showed that BDNF serum levels were significantly elevated at $\mathrm{T} 7$ than at the baseline $(\mathrm{p}<0.005)$. After that $(\mathrm{T} 14, \mathrm{~T} 21, \mathrm{~T} 30$ and T90), BDNF levels were comparable to baseline values (T0) (Figure 3).

\section{DISCUSSION}

This study was performed to investigate whether a motor rehabilitation protocol based on physiotherapy and wii balance may have therapeutic effect and/or induce changes in BDNF serum levels in a group of hospitalized PD patients. Before and after the treatment (30 days) and in the successive follow-up (90 days from the beginning of the treatment) we evaluated PD symptoms by using the clinical scales and measured BDNF serum levels by ELISA. The results showed that at the 
end of the treatment the motor rehabilitation protocol induced an improvement of extrapyramidal signs (UPDRS III; UPDRS III - Gait and Balance items) and activities of daily living(UPDRS II; PDQ-39) and a decrease in walking slowness as measured with the 6 Minute Walking Test (6MWT).At the 90day follow-up, the improvement in extrapyramidal signs (UPDRS III; UPDRS III - Gait and Balance items) and activities of daily living(UPDRS II; PDQ-39) was still present, although at a reduced level.

Our data demonstrate that the motor rehabilitation protocol adopted in this study is able to induce an improvement of extrapyramidal and motor symptoms and activities of daily living in PD patients. These data are in line with previous studies showing that physical rehabilitation treatments are able to improve gait and balance in PD patients with a good rate of retention over time (Frazzitta et al. 2013). In addition, other studies showed that Wii Fit balance board playing improves balance and gait in PD (Mhatre et al. 2013).

The molecular mechanisms of exercise-induced changes in PD are still not known. The fact that improvements of PD symptoms persisted after 3 months from the end of the treatment may indicate that permanent physiological changes may occur in the brain of PD subjects undergoing this type of rehabilitation program. This idea is also supported by animal studies showing that exercise may induce plastic changes in the brain leading to neuroprotective effects and reorganization of the compromised neuronal pathways (Kleim et al.2003). In mouse PD models these effects are also associated to a slow of disease progression and neurorestoration (Lau et al. 2011). Nonetheless, it cannot be excluded that persistence of improvements of PD motor symptom may be due to the continuation of training at home in the successive follow-up. If this is the case, it is possible that physiological changes observed are more linked to the effect of training on muscle strength, rather than plastic brain changes. Supporting this hypothesis, we found that BDNF is transiently elevated after 7 days from the beginning of the treatment and gradually return to baseline levels after that time. This finding is partially in line with the data obtained with a comparable protocol where an increase in BDNF levels in early stage PD subjects 
versus placebo was observed after 10 days and remained increased throughout the treatment period (Frazzitta et al. 2014). The reason for this difference is not clear. It is possible that the different disease stages of PD subjects have influenced the results. In addition, in that study PD subjects were administered rasagiline, a medication known to increase BDNF levels (Maruyama and Naoi 2013). Therefore it is possible that sustained BDNF levels over time may be caused by exposure to such medication. Thus, the present data contribute to clarify this possible confounding factor.

Whether this temporary increase in BDNF may have beneficial effects in PD patients is not clear. It is known that neuronal pathway involved in PD manifestation is the progressive degeneration of the dopaminergic neurons in the substantia nigra (Michel et al. 2013). BDNF is a potent trophic factor for these neurons (Hyman et al 1991) and is involved in synaptic plasticity (Gómez-Palacio-Schjetnan and Escobar 2013). Its potential involvement in PD is supported by the fact that PD patients are characterized by low levels of circulating BDNF (Scalzo et al. 2010) and antiparkinsonian drugs may increase these levels in PD mouse models (Gyárfás et al. 2010). Moreover, similar treatments, such as physical exercise as well as environmental enrichment, may upregulate BDNF in the brain (Gelfo et al. 2011; Kleim et al. 2003) and confer resistance to neurodegeneration in PD rodent models (Lau et al. 2011). Nonetheless, the fact that BDNF increase is not sustained over time suggests caution on the possibility that beneficial effects of a rehabilitation program are associated with changes in BDNF neuronal synthesis. Other factors besides the physiological effects of strength training on the muscle itself (optimization of the mitochondrial capacity to produce ATP, increase mitochondria size and numbers, optimized use of energetic substrate, adaptation of the muscle type) (Barbieri et al. 2015) may contribute to explain these data, such as influence on motor learning and cardiorespiratory functions (Abbruzzese et al. 2015).

On the other hand, these results are also in line with other studies showing the absence of long-lasting BDNF response to acute exercise or training (i.e. permanently increased basal peripheral BDNF 
concentration) in healthy subjects or persons with a chronic disease or disability (Knaepen et al. 2010). Thus it seems that exercise and/or training temporarily elevate basal BDNF and perhaps upregulate cellular processing of BDNF (i.e. synthesis, release, absorption and degradation). Subsequently, more BDNF could be released into the blood circulation which may, in turn, be absorbed more efficiently by central and/or peripheral tissues where it could induce a cascade of neurotrophic and neuroprotective effects. It remains unclear whether a temporary increase may be beneficial to the damaged dopaminergic neurons. In vitro studies suggest that cellular responses to BDNF may differ markedly depending on how BDNF is delivered. In cultured rat hippocampal neurons, acute and gradual increases in BDNF elicited transient and sustained activation of TrkB receptor and its downstream signaling, thus promoting neurite elongation and spine head enlargement, whereas sustained TrkB activation (as in case of permanent increase of BDNF levels) facilitated neurite branch and spine neck elongation ( $\mathrm{Ji}$ et al. 2010). These data, at least in animal models, suggest that even a temporary increase in BDNF levels may have beneficial effects in neurons. However, other studies are necessary to determine whether a transient BDNF increase in serum may contribute to the positive effects of physical training in PD patients. Furthermore, there are some limitation to our data interpretation. First, we are not measuring brain levels of BDNF although a correlation between central and peripheral BDNF levels has been suggested in animal models (Karege et al. 2002) and humans (Ziebell et al. 2012). It was demonstrated that BDNF can cross the blood brain barrier (Panet al. 1998; Poduslo and Curran 1996) in both directions, via a high capacity saturable transporter system (Pan et al.1998). Furthermore, it was reported that the BDNF level in the brain correlates with the serum BDNF concentration (Karege et al. 2002), suggesting that blood BDNF levels may reflect brain levels (Lommatzsch et al. 2005). Second, there are evidences that circulating BDNF may originate both from central and peripheral sources (Rasmussen et al. 2009). However, it was recently reported that during physical exercise the increase in BDNF concentration in humans was due to an enhanced release of 
BDNF from the brain (Rasmussen et al. 2009). Third, the number of subjects is relatively small, given to the difficulty to recruit comparable subjects in term of age and disease stage in such programs of rehabilitation. Thus, the results should be interpreted with caution and need confirmation in larger samples. Fourth, in this study only rigid-akinetic PD subjects were investigated and thus we do not know whether this rehabilitation protocol may be also effective in other clinical profiles of PD, such us unilateral-tremor kind.

In addition, the influence of stressful conditions due to hospitalization on BDNF data cannot be excluded, although all patients were highly collaborative before and during the study time.

In conclusion, this study demonstrates that a rehabilitation protocol may produce improvements of motor symptoms in patients affected by PD. Moreover, continuation of training with home exercises by PD patients may contribute to make these motor improvements stable over time. This study also shows that BDNF serum levels are elevated early during the course of the treatment. These findings support the notion that non pharmacological approaches may represent a useful adjuvant therapeutic strategy in the treatment of neurodegenerative disorders, maybe by inducing temporary changes in neuroprotective molecules.

\section{ACKNOWLEDGMENTS}

Supported by the Italian Ministry of Health.

\section{CONFLICT OF INTEREST}

The authors confirm that this article content has no conflicts of interest. 


\section{References}

Abbruzzese, G., Marchese, R., Avanzino, L., and Pelosin, E. 2015. Rehabilitation for Parkinson's disease: Current outlook and future challenges. Parkinsonism Relat. Disord., pii: S1353-8020(15): 00380-00386. doi: 10.1016/j.parkreldis.2015.09.005. PMID: 26360239.

Ali, S.F., Binienda, Z.K., and Imam, S.Z. 2011. Molecular aspects of dopaminergic neurodegeneration: gene-environment interaction in parkin dysfunction. Int. J. Environ. Res. Public. Health, 8(12): 4702-4713. doi: 10.3390/ijerph8124702. PMID: 22408597.

Angelucci, F., Ricci, V., Spalletta, G., Pomponi, M., Tonioni, F., Caltagirone, C., et al. 2008. Reduced serum concentrations of nerve growth factor, but not brain-derived neurotrophic factor, in chronic cannabis abusers. Eur Neuropsychopharmacol., 18(12):882-887. doi: 10.1016/j.euroneuro.2008.07.008. PMID: 18774699.

Barbieri, E., Agostini, D., Polidori, E., Potenza, L., Guescini, M., Lucertini, F., et al. 2015. The pleiotropic effect of physical exercise on mitochondrial dynamics in aging skeletal muscle. Oxid. Med. Cell. Longev., 2015: 917085. doi: 10.1155/2015/917085. PMID: 25945152.

Bekinschtein, P., Oomen, C.A., Saksida, L.M., and Bussey, T.J. 2011. Effects of environmental enrichment and voluntary exercise on neurogenesis, learning and memory, and pattern separation: BDNF as a critical variable? Semin. Cell. Dev. Biol., 22(5): 536-542. doi: 10.1016/j.semcdb.2011.07.002. PMID: 21767656.

Combs, S.A., Diehl, M.D., Filip, J., and Long, E. 2014. Short-distance walking speed tests in people with Parkinson disease: reliability, responsiveness, and validity. Gait Posture, 39(2): 784-788. doi: 10.1016/j.gaitpost.2013.10.019. PMID: 24246801. 
El-Tamawy, M.S., Abd-Allah, F., Ahmed, S.M., Darwish, M.H., and Khalifa, H.A. 2014. Aerobic exercises enhance cognitive functions and brain derived neurotrophic factor in ischemic stroke patients. NeuroRehabilitation, 34(1): 209-213. doi: 10.3233/NRE-131020. PMID: 24284463.

Folstein, M.F., Folstein, S.E., and McHugh, P.R. 1975. "Mini-mental state". A practical method for grading the cognitive state of patients for the clinician. J. Psychiatr. Res., 12(3): 189-198. PMID: 1202204.

Frazzitta, G., Balbi, P., Maestri, R., Bertotti, G., Boveri, N., and Pezzoli, G. 2013. The beneficial role of intensive exercise on Parkinson disease progression. Am. J. Phys. Med. Rehabil., 92(6): 523532. doi: 10.1097/PHM.0b013e31828cd254. PMID: 23552330

Frazzitta, G., Bertotti, G., Uccellini, D., and Maestri, R. 2010. Parkinson's disease rehabilitation: a pilot study with 1 year follow up. Mov. Disord., 25(11): 1762-1763. doi: 10.1002/mds.23316. PMID: 20589873.

Frazzitta, G., Maestri, R., Ghilardi, M.F., Riboldazzi, G., Perini, M., Bertotti, G., et al. 2014. Intensive Rehabilitation Increases BDNF Serum Levels in Parkinsonian Patients: A Randomized Study. Neurorehabil. Neural Repair, 28(2): 163-168. doi: 10.1177/1545968313508474. PMID: 24213955. Frim, D.M., Uhler, T.A., Galpern, W.R., Beal, M.F., Breakefield, X.O., and Isacson, O. 1994. Implanted fibroblasts genetically engineered to produce brain-derived neurotrophic factor prevent 1-methyl-4-phenylpyridinium toxicity to dopaminergic neurons in the rat. Proc. Natl. Acad. Sci. U.S.A., 91(11): 5104-5108. PMID: 8197193.

Garcia-Ruiz, P.J., Martinez Castrillo, J.C., Alonso-Canovas, A., Herranz Barcenas, A., Vela, L., Sanchez Alonso, P., et al. 2014. Impulse control disorder in patients with Parkinson's disease under dopamine agonist therapy: a multicentre study. J. Neurol. Neurosurg. Psychiatry, 85(8): 840-844. doi: 10.1136/jnnp-2013-306787. PMID: 24434037. 
Gazewood, J.D., Richards, D.R., and Clebak, K. 2013. Parkinson disease: an update. Am. Fam. Physician, 87(4): 267-273. PMID: 23418798.

Gelb, D.J., Oliver E., Gilman S. 1999. Diagnostic criteria for Parkinson disease. Arch. Neurol., 56(1): 33-39. doi:10.1001/archneur.56.1.33. PMID: 9923759.

Gelfo, F., Cutuli, D., Foti, F., Laricchiuta, D., De Bartolo, P., Caltagirone, C., et al. 2011. Enriched environment improves motor function and increases neurotrophins in hemicerebellar lesioned rats. Neurorehabil. Neural Repair, 25(3): 243-252. doi: 10.1177/1545968310380926. PMID: 20966156.

Gómez-Palacio-Schjetnan A, and Escobar ML. 2013. Neurotrophins and synaptic plasticity. Curr. Top. Behav. Neurosci., 15: 117-136. doi: 10.1007/7854_2012_231. PMID: 23519767.

Guyatt, G.H., Sullivan, M.J., Thompson, P.J., Fallen, E.L., Pugsley, S.O., Taylor ,D.W., et al. 1985. The 6-minute walk: a new measure of exercise capacity in patients with chronic heart failure. Can. Med. Assoc. J., 132(8): 919-923. PMID: 3978515.

Gyárfás, T., Knuuttila, J., Lindholm, P., Rantamäki, T., and Castrén, E. 2010. Regulation of brainderived neurotrophic factor (BDNF) and cerebral dopamine neurotrophic factor (CDNF) by antiparkinsonian drug therapy in vivo. Cell. Mol. Neurobiol., 30(3): 361-368. doi: 10.1007/s10571009-9458-3. PMID: 19777340.

Huot, P., Johnston, T.H., Koprich, J.B., Fox, S.H., and Brotchie, J.M. 2013. The pharmacology of LDOPA-induced dyskinesia in Parkinson's disease. Pharmacol. Rev., 65(1): 171-222. doi: 10.1124/pr.111.005678. PMID: 23319549.

Hyman, C., Hofer, M., Barde, Y.A., Juhasz, M., Yancopoulos, G.D., Squinto, S.P., et al. 1991. BDNF is a neurotrophic factor for dopaminergic neurons of the substantia nigra. Nature, 350(6315): 230232. PMID: 2005978. 
Ji, Y., Lu, Y., Yang, F., Shen, W., Tang, T.T., Feng, L., et al. 2010. Acute and gradual increases in BDNF concentration elicit distinct signaling and functions in neurons. Nat. Neurosci., 13(3): 302309. doi: 10.1038/nn.2505. PMID: 20173744.

Karege, F., Schwald, M., and Cisse, M. 2002. Postnatal developmental profile of brain-derived neurotrophic factor in rat brain and platelets. Neurosci. Lett., 328(3): 261-264. PMID: 12147321.

Kleim, J.A., Jones, T.A., and Schallert, T. 2003. Motor enrichment and the induction of plasticity before or after brain injury. Neurochem. Res., 28(11): 1757-1769. PMID: 14584829.

Knaepen, K., Goekint, M., Heyman, E.M,. and Meeusen, R. 2010. Neuroplasticity - exercise-induced response of peripheral brain-derived neurotrophic factor: a systematic review of experimental studies in human subjects. Sports Med., 40(9): 765-801. doi: 10.2165/11534530-00000000000000. PMID: 20726622.

Lau, Y.S., Patki, G., Das-Panja, K., Le, W.D., and Ahmad, S.O. 2011. Neuroprotective effects and mechanisms of exercise in a chronic mouse model of Parkinson's disease with moderate neurodegeneration. Eur. J. Neurosci., 33(7): 1264-1274. doi: 10.1111/j.1460-9568.2011.07626.x. PMID: 21375602.

Levivier, M., Przedborski, S., Bencsics, C., and Kang, U.J. 1995. Intrastriatal implantation of fibroblasts genetically engineered to produce brain-derived neurotrophic factor prevents degeneration of dopaminergic neurons in a rat model of Parkinson's disease. J. Neurosci., 15(12): 7810-7820. PMID: 8613721.

Lommatzsch, M., Zingler, D., Schuhbaeck, K., Schloetcke, K., Zingler, C., Schuff-Werner, P., et al. 2005. The impact of age, weight and gender on BDNF levels in human platelets and plasma. Neurobiol. Aging, 26(1): 115-123. PMID: 15585351.

Maruyama, W., and Naoi, M. 2013. "70th Birthday Professor Riederer" induction of glial cell linederived and brain-derived neurotrophic factors by rasagiline and (-)deprenyl: a way to a disease- 
modifying therapy? J. Neural Transm., 120(1): 83-89. doi: 10.1007/s00702-012-0876-x. PMID: 22892822.

McCullough, M.J., Gyorkos, A.M., and Spitsbergen, J.M. 2013. Short-term exercise increases GDNF protein levels in the spinal cord of young and old rats. Neuroscience, 240: 258-268. doi: 10.1016/j.neuroscience.2013.02.063. PMID: 23500094.

Mehrholz, J., Friis, R., Kugler, J., Twork, S., Storch, A., and Pohl, M. 2010. Treadmill training for patients with Parkinson's disease. Cochrane Database Syst. Rev., 2010(1): CD007830. doi: 10.1002/14651858.CD007830. PMID: 20091652.

Mhatre, P.V., Vilares, I., Stibb, S.M., Albert, M.V., Pickering, L., Marciniak, C.M., et al. 2013. Wii Fit balance board playing improves balance and gait in Parkinson Disease. PM\&R, 5(9): 769-777. doi: 10.1016/j.pmrj.2013.05.019. PMID: 23770422.

Michel, P.P., Toulorge, D., Guerreiro, S., and Hirsch E.C. 2013. Specific needs of dopamine neurons for stimulation in order to survive: implication for Parkinson disease. FASEB J., 27(9): 3414-3423. doi: 10.1096/fj.12-220418. PMID: 23699175.

Mogi, M., Togari, A., Kondo, T., Mizuno, Y., Komure, O., Kuno, S., et al. 1999. Brain-derived growth factor and nerve growth factor concentrations are decreased in the substantia nigra in Parkinson's disease. Neurosci. Lett., 270(1): 45-48. PMID: 10454142.

Movement Disorder Society Task Force on Rating Scales for Parkinson's Disease. 2003. The Unified Parkinson's Disease Rating Scale (UPDRS): status and recommendations. Mov. Disord., 18(7): 738-750. PMID: 12815652.

Pan, W., Banks, W.A., Fasold, M.B., Bluth, J., and Kastin, A.J. 1998. Transport of brain-derived neurotrophic factor across the blood-brain barrier. Neuropharmacology, 37(12): 1553-1561. PMID: 9886678. 
Parain, K., Murer, M.G., Yan, Q., Faucheux, B., Agid, Y., Hirsch, E., et al. 1999. Reduced expression of brain-derived neurotrophic factor protein in Parkinson's disease substantia nigra. Neuroreport, 10(3): 557-561. PMID: 10208589.

Peppe, A., Chiavalon, C., Pasqualetti, P., Crovato, D., and Caltagirone, C. 2007. Does gait analysis quantify motor rehabilitation efficacy in Parkinson's disease patients? Gait and Posture, 26(3): 452-462. PMID: 17240143.

Pereira, D.S., de Queiroz, B.Z., Miranda, A.S., Rocha, N.P., Felício, D.C., Mateo, E.C., et al. 2013. Effects of physical exercise on plasma levels of brain-derived neurotrophic factor and depressive symptoms in elderly women--a randomized clinical trial. Arch. Phys. Med. Rehabil., 94(8): 14431450. doi: 10.1016/j.apmr.2013.03.029. PMID: 23602881.

Peto, V., Jenkinson, C., Fitzpatrick, R., and Greenhall, R. 1995. The development and validation of a short measure of functioning and well being for individuals with Parkinson's disease. Qual. Life Res., 4(3): 241-248. PMID: 7613534.

Poduslo, J.F., and Curran, G.L. 1996. Permeability at the blood-brain and blood-nerve barriers of the neurotrophic factors: NGF, CNTF, NT-3, BDNF. Brain Res. Mol. Brain Res., 36(2): 280-286. PMID: 8965648.

Pompeu, J.E., Mendes, F.A., Silva, K.G., Lobo, A.M., Oliveira Tde, P., Zomignani, A.P., et al. 2012. Effect of Nintendo Wii ${ }^{\text {TM}}$-based motor and cognitive training on activities of daily living in patients with Parkinson's disease: a randomised clinical trial. Physiotherapy, 98(3): 196-204. doi: 10.1016/j.physio.2012.06.004. PMID: 22898575.

Rasmussen, P., Brassard, P., Adser, H., Pedersen, M.V., Leick, L., Hart, E., et al. 2009. Evidence for a release of brain-derived neurotrophic factor from the brain during exercise. Exp. Physiol., 94(10): 1062-1069. doi: 10.1113/expphysiol.2009.048512. PMID: 19666694. 
Ricci, V., Pomponi, M., Martinotti, G., Bentivoglio, A., Loria, G., Bernardini, S., et al. 2010. Antidepressant treatment restores brain-derived neurotrophic factor serum levels and ameliorates motor function in Parkinson disease patients. J. Clin. Psychopharmacol., 30(6): 751-753. doi: 10.1097/JCP.0b013e3181fc2ec7. PMID: 21057246.

Scalzo, P., Kümmer, A., Bretas, T.L., Cardoso, F., and Teixeira, A.L. 2010. Serum levels of brainderived neurotrophic factor correlate with motor impairment in Parkinson's disease. J. Neurol., 257(4): 540-545. doi: 10.1007/s00415-009-5357-2. PMID: 19847468.

Sharma, A., Goyal, V., Behari, M., Srivastva, A., Shukla, G., and Vibha, D. 2015. Impulse control disorders and related behaviours (ICD-RBs) in Parkinson's disease patients: Assessment using "Questionnaire for impulsive-compulsive disorders in Parkinson's disease" (QUIP). Ann. Indian Acad. Neurol., 18(1): 49-59. doi: 10.4103/0972-2327.144311. PMID: 25745311.

Schmolesky, M.T., Webb, D.L., and Hansen, R.A. 2013. The effects of aerobic exercise intensity and duration on levels of brain-derived neurotrophic factor in healthy men. J. Sports Sci. Med., 12(3): 502-511. PMID: 24149158.

Tomlinson, C.L., Patel, S., Meek, C., Herd, C.P., Clarke, C.E., Stowe, R., et al. 2013. Physiotherapy versus placebo or no intervention in Parkinson's disease. Cochrane Database Syst. Rev., 2013(9): CD002817. doi: 10.1002/14651858.CD002817. PMID: 24018704.

Tsukahara, T., Takeda, M., Shimohama, S., Ohara, O., and Hashimoto, N. 1995. Effects of brainderived neurotrophic factor on 1-methyl-4-phenyl-1,2,3,6-tetrahydropyridine- induced parkinsonism in monkeys. Neurosurgery, 37: 733-741. PMID: 8559303.

Vaughan, S,. Wallis, M., Polit, D., Steele, M., Shum, D., and Morris, N. 2014. The effects of multimodal exercise on cognitive and physical functioning and brain-derived neurotrophic factor in older women: a randomised controlled trial. Age Ageing, 43(5): 623-629. doi: 10.1093/ageing/afu010. PMID: 24554791. 
Vassar, S.D., Bordelon, Y.M., Hays, R.D., Diaz, N., Rausch, R., Mao, C., et al. 2012. Confirmatory factor analysis of the motor unified Parkinson's disease rating scale. Parkinsons Dis., 2012; 2012: 719167. doi: 10.1155/2012/719167. PMID: 23133789.

Weintraub, D., Hoops, S., Shea, J.A., Lyons, K.E., Pahwa, R., Driver-Dunckley, E.D., et al. 2009. Validation of the questionnaire for impulsive-compulsive disorders in Parkinson's disease. Mov. Disord., 24(10): 1461-1467. doi: 10.1002/mds.22571. PMID: 19452562.

Wilcox, R.R. 1987. New Statistical Procedures for the Social Sciences. Erlbaum: Hillsdale, New Jersey.

Worth, P.F. 2013. How to treat Parkinson's disease in 2013. Clin. Med., 13(1): 93-96. PMID: 23472504.

Zhang, J.L., Chan, P. 2012. Reliability and validity of PDQ-39: a quality-of-life measure for patients with PD in China. Qual. Life Res., 21(7): 1217-1221. doi: 10.1007/s11136-011-0026-1. PMID: 21983714.

Ziebell, M., Khalid, U., Klein, A.B., Aznar, S., Thomsen, G., Jensen, P., et al. 2012. Striatal dopamine transporter binding correlates with serum BDNF levels in patients with striatal dopaminergic neurodegeneration. Neurobiol. Aging, 33(2): 428.e1-5. doi: 10.1016/j.neurobiolaging.2010.11.010. PMID: 21176997. 
Table 1. Demographic and clinical characteristics of Parkinson's disease patients.

\begin{tabular}{c|c|c|c|c|c|c|c|c}
\hline Patient & Age & Sex & $\begin{array}{c}\text { Hohen \& } \\
\text { Yahr } \\
\text { stadiation }\end{array}$ & PD onset & $\begin{array}{c}\text { Disease } \\
\text { duration } \\
\text { (years) }\end{array}$ & $\begin{array}{c}\text { Time on } \\
\text { medication } \\
\text { (years) }\end{array}$ & $\begin{array}{c}\text { Present therapy } \\
\text { (mg LD) }\end{array}$ & $\begin{array}{c}\text { Prevalent } \\
\text { side }\end{array}$ \\
\hline $\mathbf{1}$ & 74 & M & II & 69 & 5 & 5 & 210 & Right \\
$\mathbf{2}$ & 58 & M & II & 51 & 7 & 6 & 200 & Right \\
$\mathbf{3}$ & 63 & F & II & 53 & 10 & 9 & 610 & Right \\
$\mathbf{4}$ & 68 & F & II & 63 & 5 & 2 & 500 & Left \\
$\mathbf{5}$ & 60 & M & II & 38 & 22 & 17 & 840 & Left \\
$\mathbf{6}$ & 63 & M & III & 54 & 9 & 8 & 710 & Right \\
$\mathbf{7}$ & 50 & M & III & 38 & 12 & 12 & 800.5 & Right \\
$\mathbf{8}$ & 63 & M & III & 37 & 26 & 26 & 900 & Right \\
$\mathbf{9}$ & 66 & M & II & 56 & 10 & 10 & 360 & Right \\
\hline
\end{tabular}

PD: Parkinson's Disease; M: male; F: female; LD: levodopa;R: right; L: left. 


\section{Figure legends}

Table 1. Demographic and clinical characteristics of Parkinson's disease patients. PD: Parkinson's Disease; M: male; F: female; mg LD: milligram Levodopa;R: right; L: left.

Figure 1: Overview of the experimental design. UPDRS: Unified Parkinson's Disease Rating Scale part II (Activities of daily living) and III (Motor examination); PDQ-39: Parkinson's Disease Questionnaire; 6MWT: 6 Minute Walking Test.

Figure 2: Clinical assessments at the beginning (T0), the end of the treatment (T30) and 3 months followup (T90). Data are represented as means \pm standard deviation. Asterisk $\left(^{*}\right)$ indicates significant difference between the groups. $(*=p<0.05 ; * *=p<0.01)$.

Figure 3: BDNF serum levels at the beginning (T0), $7^{\text {th }}(\mathrm{T} 7), 14^{\text {th }}(\mathrm{T} 14), 21^{\text {st }}(\mathrm{T} 21)$, and $30^{\text {th }}$ (T30) days of the treatment and at 3 months follow-up (T90). Data are represented as means \pm standard deviation. Asterisk $(*)$ indicates significant difference between the groups. $(* * *=p<0.005)$. 


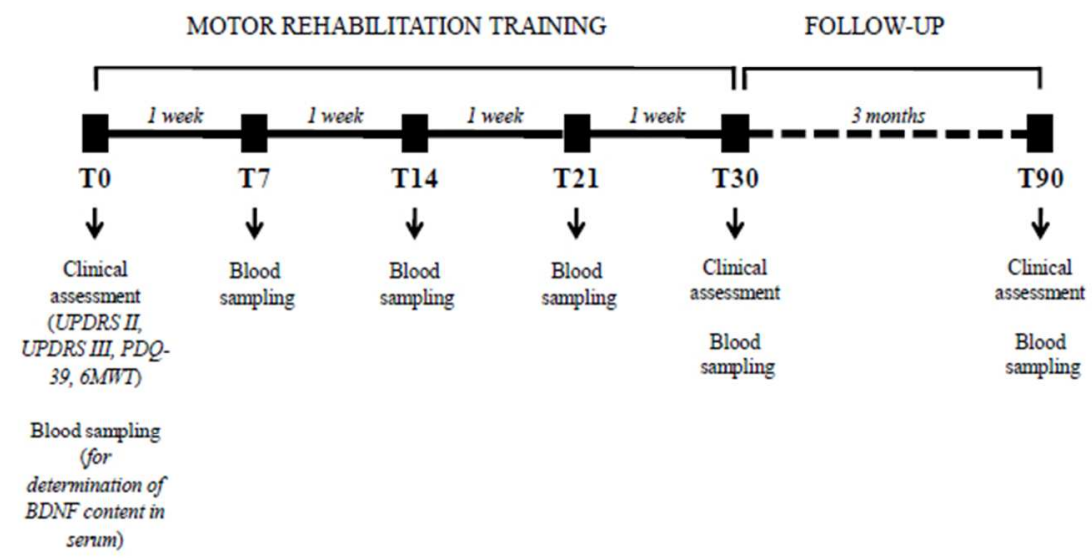

Figure 1: Overview of the experimental design. UPDRS: Unified Parkinson's Disease Rating Scale part II (Activities of daily living) and III (Motor examination); PDQ-39: Parkinson's Disease Questionnaire; 6MWT: 6 Minute Walking Test.

$63 \times 41 \mathrm{~mm}(300 \times 300$ DPI $)$ 

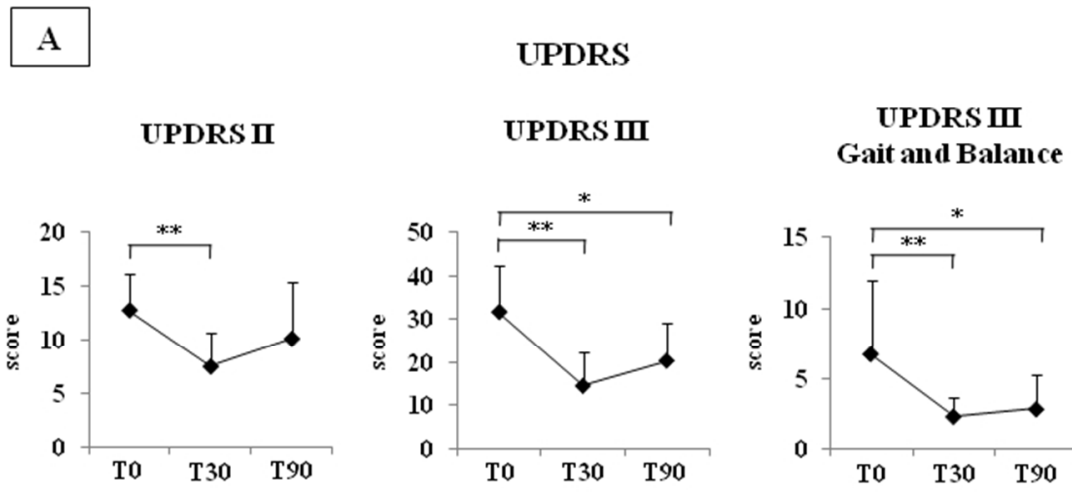

\section{B}

PDQ-39
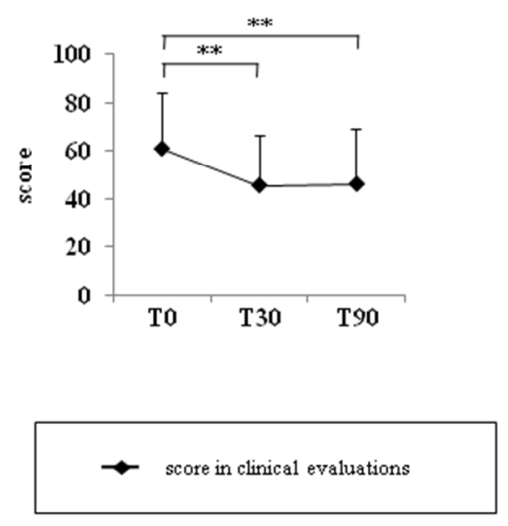
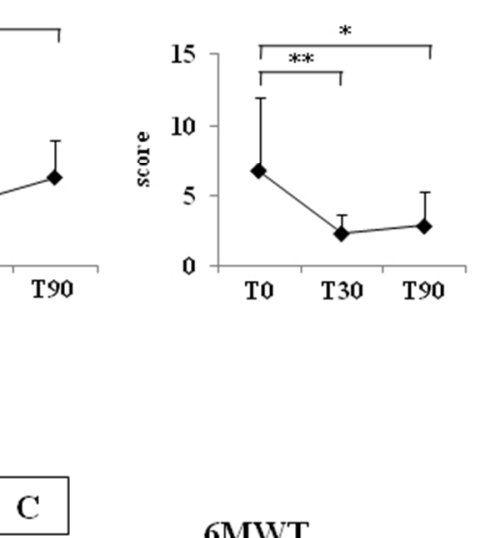

6MWT

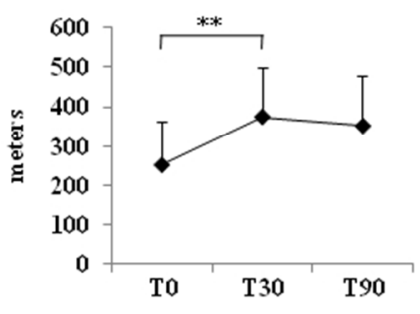

Data are represented as means \pm standard deviation.

Figure 2: Clinical assessments at the beginning (TO), the end of the treatment (T30) and 3 months follow-up (T90). Data are represented as means \pm standard deviation. Asterisk $(*)$ indicates significant difference between the groups. $(*=p<0.05 ; * *=p<0.01)$. $60 \times 81 \mathrm{~mm}(300 \times 300 \mathrm{DPI})$ 


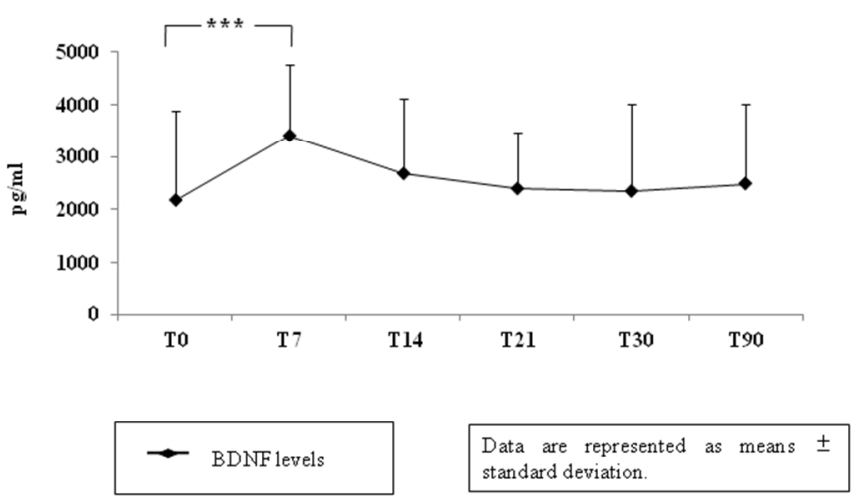

Figure 3: BDNF serum levels at the beginning (T0), 7th (T7), 14th (T14), 21st (T21), and 30th (T30) days of the treatment and at 3 months follow-up (T90). Data are represented as means \pm standard deviation. Asterisk $(*)$ indicates significant difference between the groups. $(* * *=p<0.005)$.

$81 \times 60 \mathrm{~mm}(300 \times 300 \mathrm{DPI})$ 